\title{
PERFIL DA COMERCIALIZAÇÃO DA QUETIAPINA E SUAS IMPLICAÇÕES CLÍNICAS NO TRATAMENTO DO TRANSTORNO DO HUMOR BIPOLAR
}

Willyan Junior Gonçalves Adriele Laurinda Silva ${ }^{3}$

\section{RESUMO}

O Transtorno do Humor Bipolar (THB) é caracterizado por mudanças importantes de humor entre mania e depressão, afetando cerca de $1,6 \%$ da população. A quetiapina é uma antipsicótico atípico sendo o primeiro fármaco a ganhar indicação específica para a depressão bipolar na Europa. Assim este estudo objetivou demonstrar o perfil de comercialização do fármaco Quetiapina em uma Drogaria Particular de Patos de Minas e realizar uma revisão bibliográfica desse fármaco, abordando seu mecanismo de ação e demais implicações clínicas no tratamento do THB. O delineamento do estudo foi Transversal e Retrospectivo do tipo Estudo da Utilização de Medicamentos Quantitativo bem como uma Revisão Bibliográfica sobre este fármaco no THB. Coletaram-se dados de vendas de uma Drogaria Particular do centro de Patos de Minas no período de setembro de 2014 a abril de 2015 de todas as diferentes apresentações comerciais da Quetiapina. O Consumo Médio Mensal foi de 88,15 unidades, incluindo todas as apresentações farmacêuticas, sendo que as vendas nos meses de outubro (120), novembro (101) e dezembro (101) foram superiores à média, totalizando 705 vendas neste período de 8 meses. As apresentações do fármaco genérico foram as que possuíram a maior venda $(73 \%)$ em relação aos similares $(26 \%)$ e aos de Referência (1\%), provavelmente pelo menor preço bem como a maior prescrição do fármaco pelo nome genérico. A Quetiapina atua em três sistemas neurobiológicos do transtorno: via dopaminérgica, serotoninérgica $\mathrm{e}$ noradrenérgica, sendo um tratamento eficaz para este transtorno. Conclui-se que o fármaco quetiapina possui uma boa relação risco/benefício em relação a outros fármacos no manejo do THB e que suas vendas foram maiores no final do ano, bem como houve preferência pelas apresentações genéricas.

Palavras chaves: Quetiapina, Transtorno do humor bipolar, Estudo da Utilização de Medicamentos.

\section{ABSTRACT}

The Bipolar Disorder (BD) is characterized by major mood swings between mania (euphoria) and depression, affecting about $1.6 \%$ of the population. Quetiapine is an atypical antipsychotic with the first drug to gain specific indication for bipolar depression in Europe. This study aimed to demonstrate the marketing profile of Quetiapine drug in a drugstore

\footnotetext{
${ }^{3}$ Endereço eletrônico de contato: adriele_silva147@hotmail.com
}

Volume 2, Número 1 - Maio, 2016. 
Private Patos de Minas and conduct a literature review of the drug by addressing its mechanism of action and other clinical implications in the treatment of BD. The study design was cross-sectional retrospective study of the use of the type of Quantitative Drug well as a Literature Review about Quetiapine fumarate and its clinical responsibilities in the management of BD. They were collected sales record data from a private drugstore in Patos de Minas center from September 2014 to April 2015 all of the different commercial presentations of Quetiapine. The Average Monthly Consumption was 88.15 units, including all strengths and pharmaceutical forms, with sales in October (120) November (101) and December (101) were above average, totaling 705 sales in this period 8 months. The presentations of the generic drug were those who owned the largest sale $(73 \%)$ compared to similar $(26 \%)$ and the Reference $(1 \%)$, probably at the lowest price and the highest prescription drug under the generic name. Therefore Quetiapine acts neurobiological disorder in three systems: via dopaminergic, serotonergic and noradrenergic being an effective treatment for this disorder. We conclude that the drug quetiapine has a risk/benefit over other drugs in the management of THB and its sales were higher at the end of the year, and were the preferred generic presentations.

Key words: Quetiapine, Bipolar Disorder, Study of the Use of Medicines.

\section{INTRODUÇÃO}

Os transtornos do humor não são um assunto novo já que alterações nas emoções têm sido descritas em manuscritos tanto da Grécia como da Pérsia antiga. Estes transtornos são alterações do pensamento, emoções e o comportamento de um indivíduo afetando seu dia a dia, podendo apresentar somente alguns sintomas em vez de um episódio como um todo. ${ }^{(1,2)}$

Também chamado de transtorno afetivo bipolar (TAB), são transtornos crônicos caracterizados por mudanças importantes de humor entre mania (euforia) e depressão, afetando cerca de $1,6 \%$ da população. Tem importante impacto na vida do paciente, visto que pode ocasionar danos funcionais significativos, como problemas para se cuidar, condutas inadequadas e problemas de se relacionar. ${ }^{(3,4)}$

O tratamento deste transtorno consiste em estabilizadores do humor, antidepressivos e antipsicóticos atípicos como o Lítio, Carbamazepina, Ácido Valpróico/Valproato, Gabapentina, Lamotrigina, Topiramato, Oxcarbamazepina, Risperidona, Ziprazidona, Quetiapina, Olanzapina e a Eletroconvulsoterapia. ${ }^{(5)}$

A quetiapina é o primeiro fármaco a ganhar indicação específica para a depressão bipolar na Europa, onde atua em três sistemas neurotransmissores neurobiológicos na depressão bipolar. Na via dopaminérgica, levando a um aumento da liberação da dopamina pré-frontal por um antagonismo dos receptores $5-\mathrm{HT}_{2} \mathrm{~A}$, agonismo parcial do adrenorreceptor 5- $\mathrm{HT}_{1} \mathrm{~A}$ e antagonismo do adrenorreceptor $\alpha 2$. $\mathrm{Na}$ comunicação Volume 2, Número 1 - Maio, 2016. 
serotoninérgica por elevação da densidade dos receptores $5-\mathrm{HT}_{1} \mathrm{~A}$ no córtex pré-frontal e por antagonismo dos adrenorreceptores $5-\mathrm{HT}_{2} \mathrm{~A}$ e $\alpha 2$. $\mathrm{E}$ a norquetiapina, que é o principal metabólito ativo da quetiapina, ativando um antagonista $5-\mathrm{HT}_{2} \mathrm{C}$ que é um potente inibidor do transportador da norepinefrina (NET), aumentando a norepinefrina na sinapse. ${ }^{(6)}$

Apesar de o tratamento farmacológico ser fundamental no THB, precisam incluir abordagens sócias educativas a respeito da doença tanto para o paciente quanto para sua família, com informações sobre a doença, drogas empregadas, aspectos nutricionais, atividades físicas, impactos sociais, estimulando, o paciente a conhecer mais sobre a doença e identificar os sinais precoce do começo de uma ocorrência de mania, depressão, ou misto.(5)

O objetivo geral foi demonstrar o perfil de comercialização da quetiapina e também realizar uma revisão literária desse fármaco, abordando o mecanismo de ação e suas demais implicações clínicas para o transtorno do humor bipolar.

Os objetivos específicos foram abordar o mecanismo de ação e demais implicações clínicas do Fumarato de Quetiapina, como reações adversas, dosagens terapêuticas e interações medicamentosas clinicamente relevantes, comparar o perfil de venda das diferentes dosagens do fumarato de quetiapina, avaliar quantitativamente e qualitativamente a comercialização do fumarato de quetiapina em uma farmácia de Patos de Minas e Integrar o farmacêutico na equipe multiprofissional de assistência ao paciente com transtorno de humor bipolar.

É um medicamento neuroléptico relativamente novo no mercado para o tratamento do transtorno do humor bipolar dentre outras indicações, portanto o tema abordado pretende trazer informações quanto ao seu uso racional. Esse tipo de transtorno é uma doença cada vez melhor diagnosticada e estudos demonstram que as indicações de quetiapina para pacientes vêm aumentando.

\section{METODOLOGIA}

Esta pesquisa trata- se de um Estudo Farmacoepidemiológico Transversal e Retrospectivo do tipo Estudo da Utilização de Medicamentos na forma quantitativo e qualitativo. Se trata também de uma revisão bibliográfica sobre o Fumarato de Quetiapina e suas atribuições clínicas no manejo do Transtorno do Humor Bipolar

Os Estudos de Utilização de Medicamentos foram definidos pela Organização Mundial de Saúde, em 1977, como "a comercialização, a distribuição, prescrição e o uso de

Volume 2, Número 1 - Maio, 2016. 
medicamentos em uma sociedade, com ênfase especial sobre as consequências médicas, sociais e econômicos resultantes".(7)

Estudos quantitativos são aqueles cujo objetivo é quantificar o consumo de medicamentos de uma determinada região, permitindo a comparação com outras localidades ou em diferentes períodos. ${ }^{(8)}$

A pesquisa foi realizada na cidade de Patos de Minas um município com cerca de 138.710 habitantes, com uma área de $3.189,771 \mathrm{~km}^{2}$ de acordo com o IBGE 2015.

A presente pesquisa coletou dados de registro de vendas de uma Drogaria Particular localizada no centro da cidade no período de setembro de 2014 a abril de 2015 em relação ao Fármaco Quetiapina, independentemente de suas indicações clínicas.

O programa utilizado pela drogaria é Gestão Itec. Os dados foram arranjados pelos nomes comerciais: QUEOPINE ${ }^{\circledR}$; QUEROPAX ${ }^{\circledR}$; QUETROS $^{\circledR}$; SEROQUEL ${ }^{\circledR}$; SEROQUEL XRO ${ }^{\circledR}$; QUETIAPINA SANDOZ ${ }^{\circledR}$; QUETIAPIANA ACTAVIS ${ }^{\circledR}$ e unidades de vendas mensais para cada medicamento foram organizadas em gráficos através do Excel 2010 conforme mostrados nos resultados e discursão.

Realizou-se também, uma revisão bibliográfica sobre o fármaco fumarato de quetiapina e suas implicações clínicas, para isso foram utilizados artigos científicos publicados em periódicos, bases de dados, livros, monografias, dissertações e teses desde 1992 a 2013. Utilizando como palavras de busca: quetiapina, transtorno do humor bipolar e quetiapina, indicações clínicas de quetiapina, tratamento para transtorno do humor bipolar.

\section{FUMARATO DE QUETIAPINA}

O fumarato de quetiapina foi descoberto em 1984, e comercializado inicialmente pela AstraZeneca com o nome de Seroque ${ }^{\circledR}$. Teve aprovação em mais de 70 países e em 1997 foi aprovado pela Food and Drug Administration (FDA) como fármaco psicotrópico e em 2006 para tratamentos de transtornos depressivos associados a bipolar I e II, alcoolismo, estresse pós-traumático, Parkinson, Síndrome de Tourette e sedativo para tratamento de insônia e ansiedade ${ }^{9}$. E foi o primeiro fármaco a ganhar indicação específica para a depressão bipolar na Europa.(6)

Os nomes comerciais do fumarato de quetiapina são: Seroquel ${ }^{\circledR}$, Seroquel $\mathrm{XRO}^{\circledR}$, Kitapen $^{\circledR}$, Neotiapim $^{\circledR}$, Neuroquel $^{\circledR}$, Queopine $^{\circledR}$, Querok $^{\circledR}$, Querox $^{\circledR}$, Queropax $^{\circledR}$, Quetiel $^{\circledR}$, Quetipin $^{\circledR}$, Quetros ${ }^{\circledR}$. Com apresentações de comprimidos de $25 \mathrm{mg}, 100 \mathrm{mg}, 200 \mathrm{mg}$ e 300mg

Volume 2, Número 1 - Maio, 2016. 
e liberação prolongada de $50 \mathrm{mg}, 200 \mathrm{mg}$ e $300 \mathrm{mg}$ onde deve conservar a temperatura ambiente de $15^{\circ} \mathrm{C}$ a $25^{\circ} \mathrm{C}$. $^{(10)}$

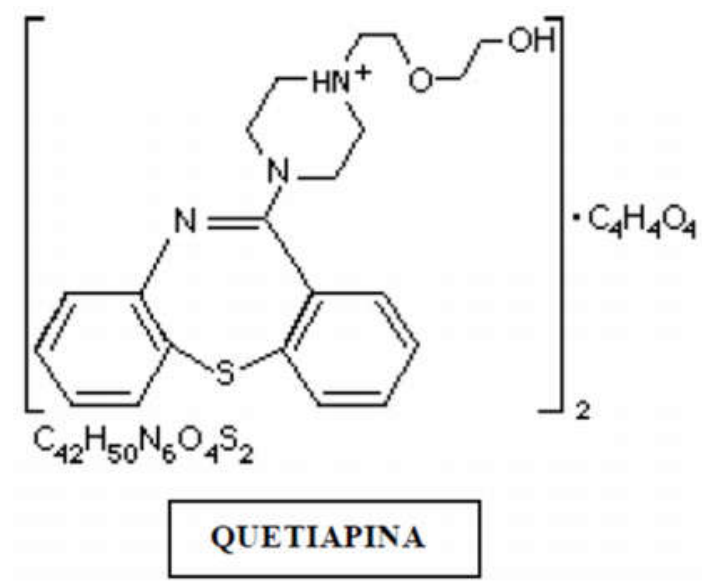

Figura 1: Estrutura molecular do fumarato de quetiapina. ${ }^{(11)}$

De acordo com a figura 1 a quetiapina, com a cadeia: 2-(2-(4-dibenzo $(b, f)$ $(1,4)$ tiazepin-11-il-1-piperazinil)etoxi)-,(E)-2-butenodioato $\quad(2: 1)(\mathrm{sal}) \quad$ ou 2-(2-(4Dibenzo(b,f)(1,4)tiazepina-11-il-1-piperazinil)etoxi)et-anol, ou também ICl 204,636, CAS RN Number 111974-72-2, é uma substância do grupo das dibenzotiazepinas, (clozapina, fluperlapina, perlapina) e às tienobenzodiazepinas (olanzapina, flumazepina), com propriedades de um antipsicótico atípico, com poucos efeitos adversos e promovendo efeito sobre a esquizofrenia. ${ }^{(12)}$

Suas indicações são para: esquizofrenia, episódios maníacos, depressão associados ao transtorno afetivo bipolar.(10)

\subsection{Mecanismo de ação}

O fármaco atua em três sistemas neurotransmissores neurobiológicos na depressão bipolar. Na via dopaminérgica, levando a um aumento da liberação da dopamina pré-frontal por um antagonismo dos receptores 5- $\mathrm{HT}_{2} \mathrm{~A}$, agonismo parcial do adrenorreceptor 5- $\mathrm{HT}_{1} \mathrm{~A}$ e antagonismo do adrenorreceptor $\alpha 2$. Na comunicação serotoninérgica por elevação da densidade dos receptores $5-\mathrm{HT}_{1} \mathrm{~A}$ no córtex pré-frontal e por antagonismo dos adrenorreceptores $5-\mathrm{HT}_{2} \mathrm{~A}$ e $\alpha 2$. $\mathrm{E}$ a norquetiapina, que é o principal

Volume 2, Número 1 - Maio, 2016. 
metabólito ativo da quetiapina, ativando um antagonista $5-\mathrm{HT}_{2} \mathrm{C}$ que é um potente inibidor do transportador da norepinefrina (NET), aumentando a norepinefrina na sinapse. ${ }^{(6)}$

É um medicamento absorvido por via oral e metabolizado no fígado, sem sofrer efeitos pela presença de alimentos, sendo seu pico plasmático cerca de 2 horas, possui um tempo de meia-vida curta sendo de 7 horas podendo ser administrado 2 a 3 vezes ao dia, onde aconselha a utilizar doses maiores no período noturno pelos seus efeitos sedativos onde recomenda doses iniciais baixas aumentando progressivamente com dose máxima de $800 \mathrm{mg} / \mathrm{dia}^{(11)}$

Podem ocorrer efeitos como: cefaléia, sonolência, ganho de peso, taquicardia, tontura, dor abdominal, boca seca, anorexia, fraqueza, tosse, aumento do colesterol, hipotireoidismo, aumento do apetite. ${ }^{(12)}$

\section{TRANSTORNO DO HUMOR BIPOLAR (THB)}

$\mathrm{Na}$ Antiguidade até início do século XIX, a melancolia e a mania eram consideradas condições totalmente diferentes, de origem física, englobando desde os transtornos mentais orgânicos à esquizofrenia.(14)

O renascer do transtorno bipolar na era moderna foi no ano de 1851 por Jean-Pierre Falret, que criou o termo "folie circulaire". Depois de 2 anos, Jules Baillarger descreveu a "folie à double forme". As duas descrições equivalem ao conceito contemporâneo de transtorno bipolar. A concordância por Kahlbaum do conceito de "folie circulaire" foi definitiva na adoção deste na Alemanha. Em seguida, houve a ampliação de sua utilização pelo continente europeu. ${ }^{(15)}$

No ano de 1966, dois trabalhos importantes fortalecem o conceito de transtorno bipolar. O de Jules Angst foi publicado em sua monografia On the Aetiology and Nosology of Depressive Disorders. E o do escrito Perris, intitulado A study of bipolar (manic-depressive) and unipolar recurrent depressive psychoses. ${ }^{(16)}$

O conceito bipolar passou a ser tratado na da década de 1970 onde Hagop Akiskal trabalhou esse conceito, sugerindo subdivisões do THB, apresentando sete tipos de transtorno ao ser ampliado o conceito ouve redução significativamente. Entretanto, o conceito de transtorno bipolar está em constante processo de evolução·(17,18,19)

\subsection{Definição}

Volume 2, Número 1 - Maio, 2016. 
O transtorno do humor bipolar é mais do que alterações de humor que as pessoas vivenciam em relação aos pontos altos e baixos da vida, pois implicam episódios de humor no qual pensamento, emoções e o comportamento alteram-se visivelmente, afetando o cotidiano. Entretanto poderá haver apenas alguns sintomas em vez de um episódio propriamente dito, ter o conhecimento dos sintomas ou episódios pode ajudar a identificar a doença. ${ }^{(20)}$

É relatado como um dos mais graves tipos de transtorno mental e abrange aspectos neuroquímicos, cognitivos, psicológicos, funcionais e socioafetivos e está coligado a altos índices de mortalidade e danos socioeconômicos onde um acompanhamento em longo prazo é fundamental É um dos quadros mais persistentes ao longo da história da medicina com formas típicas de euforia, mania e depressão que são bem caracterizadas e reconhecíveis, permitindo o diagnóstico precoce e confiável.(21-22)

A tabela 1 demonstra os tipos de episódios do transtorno do humor bipolar e suas definições e tempo mínimo para se manifestar se. "A classificação mais comum dada pela DSM-IV (Manual de Estatística e Diagnóstico de Desordens Mentais) são como TBP tipo I e tipo II, sendo que a prevalência da TBP tipo I é maior". ${ }^{21)}$

Existem vários tipos de transtorno contendo o transtorno bipolar do tipo I que contém um ou mais episódios de mania ou episódios mistos a maioria tem sintomas depressivos, o transtorno bipolar do tipo II abrange pelo menos um evento de hipomania e de depressão, ciclotimia: possui episódios de hipomania e sintomas depressivos leves que acontece na maioria dos casos num período de menos de dois anos e quando não se enquadra em algumas categorias como exemplo, pode ter sintomas leves de depressão e hipomania por um período menor de dois anos, como na ciclotimia ou pode ter episódios depressivos, mas com aumento de humor elevado leves, ou duração curta, não sendo diagnosticado como mania ou hipomania.(20)

A prevalência de THB na população geral varia de 1 a 1.6\%. A prevalência de THB do tipo I (BP I), tipo II (BP II) e Espectro Bipolar (EB). Nestes encontramos prevalências de BP I, variando entre 1 a $1,6 \%$, BP II, de 0,5 a 1,2\% e EB, de 2,4 a 2,6\%. Em uma amostra retirada da cidade de São Paulo, relata-se uma prevalência de 0,9\% para BP I, 0,2 \% para BP II e 2,1\% para EB. ${ }^{(24)}$

Volume 2, Número 1 - Maio, 2016. 
"É possível que cerca de um em cada cem adultos tenha transtorno bipolar do tipo I. Quando se inclui o transtorno bipolar do tipo II, essa prevalência aumenta para quatro em cada cem adultos".(25)

\subsection{Fisiopatologia do THB}

Já no sistema nervoso central (SNC), possui receptores que são esculpidos pela proteína G, contendo receptores noradrenérgicos, serotoninérgicos, dopaminérgicos, colinérgicos, histaminérgicos, dentre outros. O efeito desta proteína pode ser estimulado ou bloqueado, quando é estimulada, classifica como proteína $G_{s}$ e, quando é bloqueada, classifica como proteína $G_{i}$. Quando ocorre o transtorno do humor bipolar acontece um aumento nos níveis de proteína $\mathrm{G}_{\mathrm{s}}$, que ativada, modula o fluxo iônico por meio da regulação da atividade do canal iônico, possui outra função de regular a enzima adenilato ciclase, que catalisa a formação de adenosina monofosfato cíclico $\left(\mathrm{AMP}_{\mathrm{c}}\right)$ que é um segundo mensageiro importante, com função de ativar a proteína quinase A (PKA), responsável por regular os canais iônicos e fatores de transcrição. Os pacientes bipolares podem acontecer um aumento na atividade da adenilato ciclase, $\mathrm{AMP}_{\mathrm{c}}$ e $\mathrm{PKA}$, que pode estar coligado a disfunções da proteína G. Quando a proteína $G$ é ativada, o sistema de neurotransmissão usa a via do fosfoinositol, porque nela a proteína $G$ excita a fosfolipase $C$, que hidrolisa fosfolipídios da membrana, formando fosfoinositol $\left(\mathrm{PIP}_{2}\right)$ ocorrendo à criação de dois importantes segundos mensageiros, o diacilglicerol (DAG) e o inositol trifosfato $\left(\mathrm{IP}_{3}\right)$. $\mathrm{O} \mathrm{IP}_{3}$ tem receptor específico situado no retículo endoplasmático liso, que ativa a liberação de cálcio armazenado. O DAG ativa a proteína kinase $\mathrm{C}$, implicada com processos celulares que incluem secreções, expressão gênica, modulação da conduta iônica, proliferação celular entre outras. Com isso, estudos relatam que, no THB, ocorrem alterações no caminho fosfoinositol. (26)

A redução do metabolismo energético cerebral pode estar relacionada com o transtorno bipolar, como também doenças de Alzheimer, Parkinson, Huntington e isquemia cerebral pois a diminuição da produção energética no cérebro pode afetar a síntese de neurotransmissores e lipídios e falha no funcionamento da cadeia respiratória mitocondrial podendo levar a uma instantânea queda na produção de energia e morte celular. (27,28,29,30) $^{2}$

\subsection{Diagnóstico}

Volume 2, Número 1 - Maio, 2016. 
O TB é reconhecido como uma doença herdada, sendo importante avaliar a história familiar da doença. ${ }^{(31)}$

O THB é responsável por mais anos de vida perdidos do que todas as formas de câncer ou condições neurológicas maiores como epilepsia e doença de Alzheimer, principalmente, porque se inicia na juventude e cronicamente atravessa a vida do paciente. Além disso, o transtorno é ranqueado como o nono entre as principais causas de disfunção em indivíduos entre 15 e 45 anos e, em 2020, espera-se que chegue a quarta posição.(32)

Os sintomas e aspecto clínico do THB na infância e adolescência são diferentes da vida adulta, o transtorno pré-puberal pode mostrar-se como: estresse elevado, sinais de depressão e mania, ciclagem rápida, baixa recuperação dos episódios, desiquilíbrio emocional e irritabilidade durante todos os episódios, imperatividade, falar em excesso vários assuntos, não tem sono, crê ter habilidades especiais e/ou fazer coisas

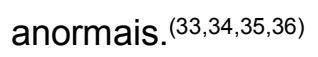

$\mathrm{Na}$ idade escolar e a adolescência no geral expõem estresse elevado, mania mista, ciclagem rápida, mania de grandeza, desiquilíbrio emocional e explosões de raiva, prevalência de fatos psicóticos tanto em crianças e adolescência, com altos percentuais de suicídio, repetência escolar, agressividade, comportamentos indisciplinares, como abuso sexual e de substâncias ilícitas. Na vida adulta o transtorno tem início entre os 18 e os 22 anos, podendo desenvolver durante a vida, podendo ter início aos 65 anos ou mais. ${ }^{(37,38,39)}$

\subsection{Tratamento}

O tratamento do transtorno do humor bipolar é dividido em três fases: a fase aguda, que é a continuação e manutenção com objetivo de tratar mania sem provocar depressão e ou melhorá-la sem causar mania. A fase de continuação tendo como alvo firmar os benefícios, diminuir os efeitos colaterais, tratar até a remissão, diminuir a probabilidade de recaída, e a manutenção que preveni a mania e ou depressão e aumenta a recuperação.(40)

\subsubsection{Tratamento farmacológico}

Volume 2, Número 1 - Maio, 2016. 
A qualidade de vida dos portadores depende do uso de medicamentos, onde um tratamento apropriado pode controlar e reduzir o transtorno, melhorando a vida do portador ou diminuir os efeitos negativos do transtorno. ${ }^{(41)}$

Podem se utilizar para este transtorno medicamentos como Estabilizadores de Humor: Lítio, Ácido Valpróico, Carbamazepina, Lamotrigina que podem diminuir os sintomas agudos, e manter o humor estável, evitando o tratamento prolongado ${ }^{42,43}$, podendo restringir o risco de suicídio.(44)

Os Antipsicóticos atípicos: Quetiapina, Olanzapina, Aripiprazol, Risperidona, Paliperidona, Amissulprida, Ziprasidona, Clozapina que controlam a mania, depressão bipolar, ansiedade, psicose, agitação e perturbações do sono. ${ }^{(42)}$

As Benzodiazepinas: Clonazepam, Diazepam, Lorazepam que podem ajudar a aliviar a ansiedade, o pânico, a agitação e insônia, sempre utilizada associada e doses elevadas são indispensáveis para obter resultados, deve utilizá-lo por períodos curtos e se necessário.(20)

Os Antidepressivos Tricíclicos como: Sertralina, Paroxetina, Fluoxetina, Escitalopram, Fluvoxamina, Amitriptilina, Imipramina, Clomipramina, Mirtazapina, Reboxetina, Bupropiona e Lamotrigina que ajudam no transtorno do humor bipolar associado a estabilizadores de humor.(45)

E a Eletroconvulsoterapia (ECT), onde o paciente é anestesiado e um estímulo elétrico atinge uma área específica do cérebro podendo ocorrer confusão temporária ou perda de memória durante ou após o tratamento. É indicada para pacientes com depressão bipolar psicótica grave, alto risco para complicações médicas, não se alimenta ou recusa alimentar e alto risco de suicídio.(46)

"A associação de Lítio, Divaproato ou antipsicóticos atípicos, especialmente a quetiapina, com antidepressivos pode ser benéfica”. Terapias adicionais como: ácidos graxos ômega- 3 que podem ajudar a diminuir a depressão em associação à medicação. ${ }^{(47,48)}$

\subsubsection{Tratamento não farmacológico}

O Tratamento Farmacológico é fundamental para estabilizar o transtorno do humor bipolar, mas necessita de outros acompanhamentos e abordagens, a Psicoeducação ou Intervenção Psicoeducativa que possibilita abordagens educativas a respeito do transtorno tanto individuais e em grupo, tanto para o paciente e sua família, com dados sobre a

Volume 2, Número 1 - Maio, 2016. 
doença, sintomas, períodos de crise, etiologia, curso e prognóstico, estresses indutores, sobre as drogas utilizadas, tempo de uso, efeitos colaterais, controles laboratoriais, nutrição, exercícios físicos, impactos sociais. Estimulando a pessoa a se informar sobre a doença, a identificar os sinais de mania, depressão, ou misto. Muitos pacientes dividem suas dúvidas, mitos e preconceitos ${ }^{(5)}$

A Psicoeducação ou Intervenção Psicoeducativa é possibilitar que as pessoas com o transtorno do humor bipolar tenham conhecimento sobre sua doença, de forma teórica e prática, para que lidem com as consequências do transtorno. Onde fornece a informação da relação entre a doença, os sintomas, a personalidade, o ambiente e os efeitos colaterais da medicação. Muitos pacientes dividem suas dúvidas, mitos e preconceitos. ${ }^{(49)}$

\section{O USO DO FUMARATO DE QUETIAPINA PARA O TRANSTORNO DO HUMOR BIPOLAR}

Entre os antipsicóticos apenas os atípicos possui efeito no transtorno do humor bipolar, a quetiapina por ser um antipsicótico atípico possui um papel fundamental no tratamento deste transtorno com o efeito bem estabelecido.(50,51)

$\mathrm{O}$ efeito da quetiapina no transtorno é devido seu metabólito, $\mathrm{N}$ esaquilquetiapina, devido sua inibição da recaptação de norepinefrina e aumento da neurotransmissão serotoninérgica pelo bloqueio de receptores $5 \mathrm{HT}_{2} \mathrm{~A}$ e por ser agonista parcial de receptores $5-\mathrm{HT}_{1} \mathrm{~A} \cdot(52)$

Para o tratamento do transtorno bipolar tipo I recomenda o uso da quetiapina associada a inibidor seletivo de recaptação de serotonina como citalopram, escitalopram, fluoxetina, fluvoxamina, paroxetina, sertralina, já para o transtorno bipolar tipo II aconselha o uso de monoterapia do fármaco.(53)

Podendo acontecer recaídas ou recorrências que em $50 \%$ dos pacientes é de três meses o tratamento de manutenção para o transtorno é extremamente importante.(54)

A finalidade do tratamento em longo prazo não abrangem somente prevenção de comportamento suicida e repetição de episódios maníacos e depressivos, mas também melhorar adesão ao tratamento e qualidade de vida e reinicia o paciente na vida social.(53)

A quetiapina é eficaz para manutenção do transtorno do humor bipolar, em monoterapia com doses de $600 \mathrm{mg} /$ dia ou também combinada com lítio e valproato.(53)

Volume 2, Número 1 - Maio, 2016. 
Quetiapina adjuntiva com estabilizador do humor quando comparado com estabilizador em monoterapia foi associado com menos episódios afetivos, menos hospitalizações, mas com mais efeitos colaterais tipo sedação, aumento de peso, hipotireoidismo e resistência à insulina. ${ }^{(55)}$

Durante a gravidez pode se fazer o uso da quetiapina ou parar no primeiro trimestre de acordo com a orientação médica, seus efeitos colaterais na gravidez podem ocorrer complicações obstétricas, diabetes gestacional e pré-eclâmpsia, para o feto macrossomia (bebês acima do peso), hipoglicemia, e durante o nascimento como fraturas e paralisia de nervos ${ }^{(56)}$

A quetiapina é indicada para crianças e adolescentes tendo efeito satisfatório em tratamento de monoterapia, um dos seus efeitos adversos é ganho de peso. Fármacos depressores do SNC como: anestésicos, hipnóticos, ansiolíticos, antidepressivos sedativos e antipsicóticos de baixa potência, devem ser evitados associados à quetiapina, pois podem potencializar ações sedativas e hipotensoras.(57-58)

Indutores enzimáticos potentes como hidantoína, carbamazepina, oxcarbazepina, barbitúricos e rifampicina podem aumentar o clearence (substância excretada na urina) da quetiapina em até 5 vezes, obrigando a ajuste de doses. ${ }^{(59)}$

A tabela 2 a seguir demonstra mais efeitos da quetiapina em relação a outros fármacos no tratamento do transtorno bipolar.

\section{RESULTADOS E DISCUSSÃO}

Os Estudos de Utilização de Medicamentos representam apoio decisivos na elaboração de politicas governamentais na área de saúde e compreender diferentes temas de investigações relativas ao uso de medicamentos. Com estes estudos é possivel compreender a demanda do uso de medicamentos e com isso verificar possibilidades de ocorrências de abuso, mau uso, uso incorreto de medicamentos e até mesmo o descumprimento da terapia.(61)

Baseado nas afirmativas deste autor se fazem necessário Estudos de Utilização de Medicamentos em cada região para assim efetuar estimativas da demanda e do perfil de comercialização de um determinado medicamento.

Volume 2, Número 1 - Maio, 2016. 
Este estudo buscou dados de vendas do fármaco Quetiapina em uma drogaria particular no Centro da cidade de Patos de Minas. Para tanto, optou-se por fazer um levantamento de vendas inicial, independente das apresentações comerciais e dos diversos fabricantes, em um período de setembro de 2014 a abril de 2015 com o objetivo de verificar se houve variações de número de unidades vendidas em cada mês e durante este período, como pode se observar na figura 1, usando apenas o nome "Quetiapina".

Tabela 2: Comparação da quetiapina e outros fármacos. ${ }^{(60)}$

\begin{tabular}{|c|c|}
\hline Quetiapina e olanzapina & $\begin{array}{l}\text { - a quetiapina produz menos distúrbios de } \\
\text { movimento (movimentos involuntários), } \\
\text { menor ganho de peso e elevação de } \\
\text { glicose. }\end{array}$ \\
\hline Quetiapina e risperidona & $\begin{array}{l}\text { - a quetiapina induz menos distúrbios de } \\
\text { movimento, diminuição do aumento de } \\
\text { prolactina (responsável por estimular a } \\
\text { produção de leite pelas glândulas } \\
\text { mamárias e o aumento das mamas), } \\
\text { aumento do colesterol. }\end{array}$ \\
\hline Quetiapina e paliperidona & $\begin{array}{l}\text { - a quetiapina possui menos efeitos } \\
\text { colaterais parkinsonianas e menores } \\
\text { aumento de prolactina e ganho de peso. }\end{array}$ \\
\hline Quetiapina e ziprasidona & $\begin{array}{l}\text { - a quetiapina induz menos efeitos } \\
\text { extrapiramidais (movimentos musculares } \\
\text { irregulares e involuntários, geralmente na } \\
\text { face), e diminuição do aumento de } \\
\text { prolactina, mas foi mais sedativa e teve } \\
\text { maior ganho de peso e aumento de } \\
\text { colesterol comparando com ziprasidona. }\end{array}$ \\
\hline
\end{tabular}

Volume 2, Número 1 - Maio, 2016. 
A figura 1 demonstra as vendas mensais de medicamentos a base de fumarato de quetiapina no período de setembro de 2014 a abril de 2015, onde no mês de setembro ocorreram 77 vendas, no mês outubro 120 vendas, no mês de novembro e dezembro 101 vendas cada, no mês de janeiro tiveram 74 vendas, no mês de fevereiro 72 vendas, o mês de março 78 vendas e o mês de abril 82 vendas, totalizando 705 vendas neste período de 8 meses. O Consumo Médio Mensal foi de 88,15 unidades. Sendo que nos meses de outubro, novembro e dezembro, as vendas ficaram acima da média e nos demais meses, abaixo dela. Podendo ser justificado pelo maior poder aquisitivo nesta época do ano, já que muitos consumidores recebem maiores benefícios financeiros como o décimo terceiro-salário.

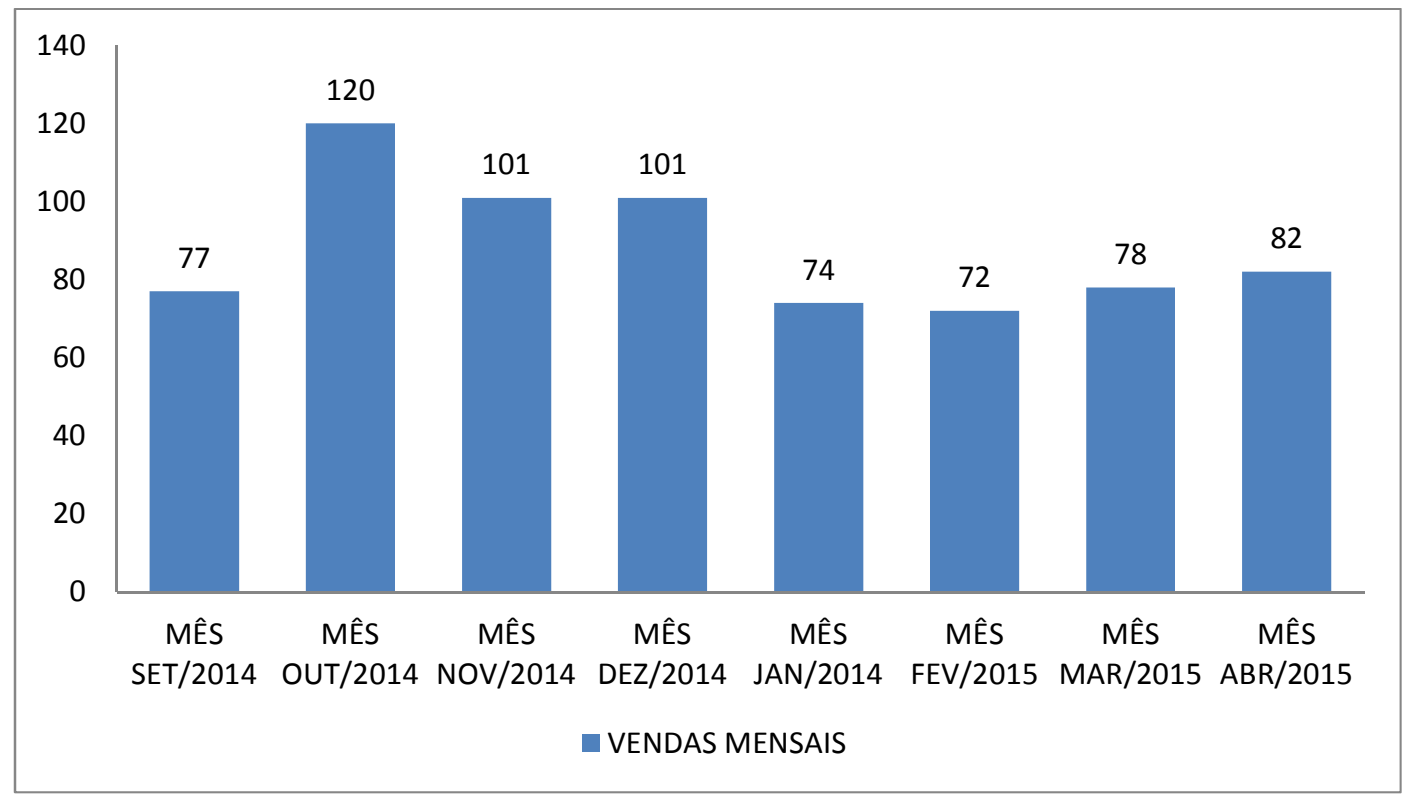

Figura 1: Vendas por unidade independente do fabricante em cada mês.

A OMS considera que uma amostra obtida em determinado momento demonstre basicamente os mesmos resultados que outra que envolva um período mais amplo. Assim espera-se que os resultados encontrados nesses 8 meses de pesquisa se repitam ao longo do ano e por isso os mesmos possam servir como fonte de informação da comercialização nessa drogaria e assim no município para definições de racionalidade e qualidade do uso dos medicamentos. ${ }^{(68)}$

Volume 2, Número 1 - Maio, 2016. 
Apesar de ser considerada uma unidade de medida mais confiável, o número de unidades vendidas tem também limitações. Em documentos postulador por Castro, em 1996, este autor afirma que devido às condições particulares de mercado, renda per capita, regulamentação e economia de cada país, estatísticas de despesas per capita ou de consumo baseado no custo podem dar uma ideia equivocada da realidade. Comparar consumos no mesmo país em diferentes períodos também acarreta problemas em casos de alta inflação ou de grande flutuação de preços. ${ }^{(63)}$

Para tanto, surgiu à necessidade de utilizar outra ferramenta para estimar o consumo de medicamentos em uma população, criou-se então, pela unidade de medida chamada DDD (Defined Daily Dose ou Dose Diária Definida), que é recomendada pela OMS, a partir de 1981, para uso em estudos de utilização de medicamentos.(63)

A DDD é definida pelo WHO Collaborating Centre como a dose média de manutenção diária para determinado fármaco, na sua indicação principal, em adultos (a referência de peso é de $70 \mathrm{~kg}$ ). Entretanto apenas com dados de registro de vendas de um medicamento, não foi possível o cálculo de DDD, seria preciso uma pesquisa em relação ao consumidor do medicamento, o que foi inviável na realização da presente pesquisa.(62)

De acordo com os dados do sistema de vendas desta drogaria, é possível separar as vendas por fabricante e, por dosagens assim como apresentações comerciais diferentes do fármaco quetiapina, conforme apresentado na figura 2, que relaciona as diferentes apresentações comerciais com o número de unidades vendidas no período de 8 meses.

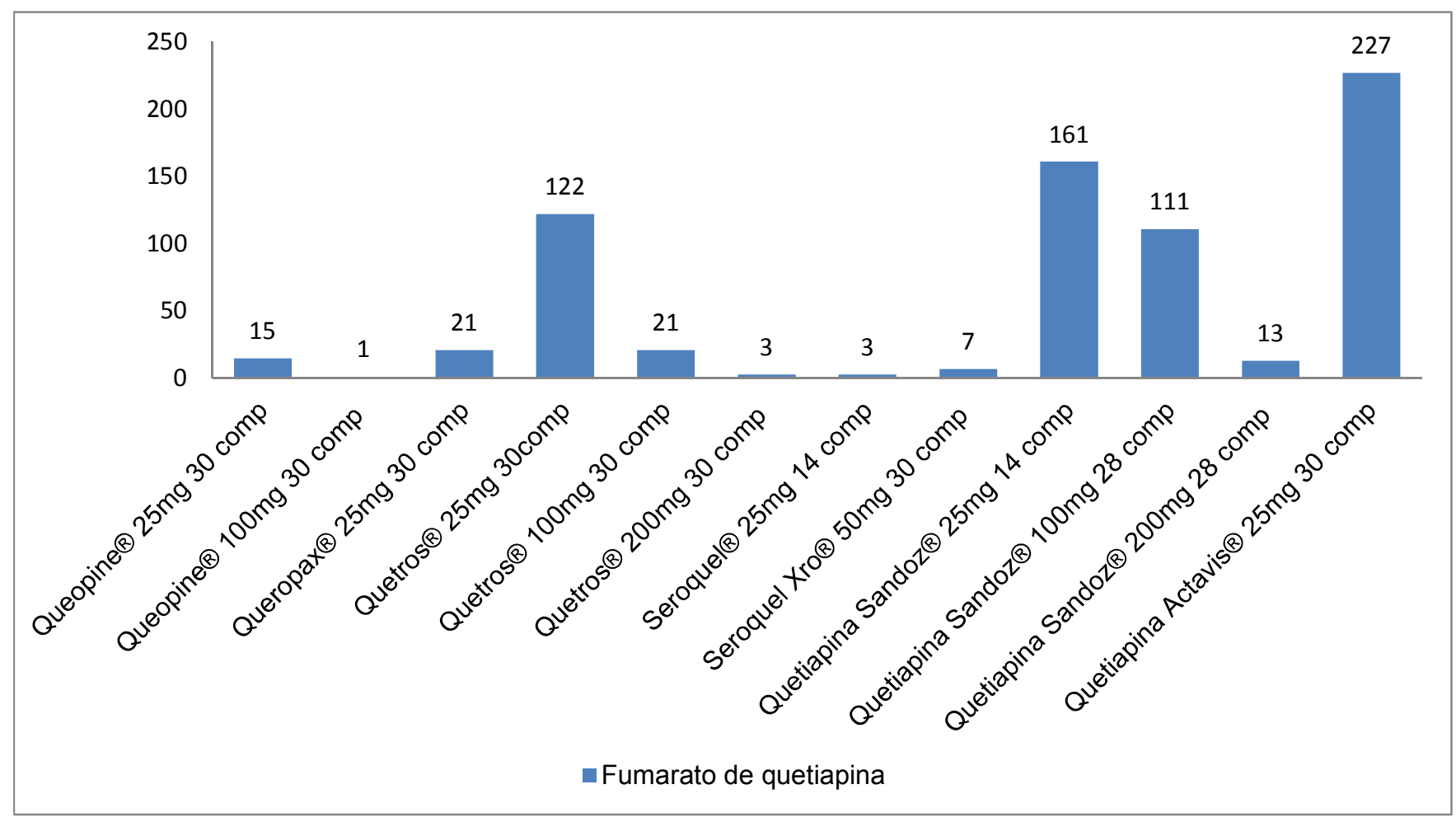


Figura 2: Vendas por fabricante, dosagens e apresentações comerciais do fármaco Quetiapina.

A figura 2 mostra a quantidade de medicamentos a base de fumarato de quetiapina vendidos no período de setembro de 2014 a abril de 2015, que são Queopine ${ }^{\circledR} 25 \mathrm{mg} 30$ comp com 15 vendas, Queopine ${ }^{\circledR} 100 \mathrm{mg} 30$ comp 1 venda, Queropax ${ }^{\circledR} 25 \mathrm{mg} 30$ comp 21 vendas, Quetros ${ }^{\circledR} 25 \mathrm{mg} 30 \mathrm{comp} 122$ vendas, Quetros ${ }^{\circledR} 100 \mathrm{mg} 30 \mathrm{comp} 21$ vendas, Quetros $^{\circledR} 200 \mathrm{mg} 30$ comp 3 vendas, Seroquel ${ }^{\circledR} 25 \mathrm{mg} 14$ comp 3 vendas, Seroquel Xro $^{\circledR}$ $50 \mathrm{mg} 30$ comp 7 vendas, Quetiapina Sandoz ${ }^{\circledR} 25 \mathrm{mg} 14$ comp 161, Quetiapina Sandoz ${ }^{\circledR}$ 100mg 28 comp 111 vendas, Quetiapina Sandoz ${ }^{\circledR} 200 \mathrm{mg} 28$ comp 13 vendas, Quetiapina Actavis ${ }^{\circledR} 25 \mathrm{mg} 30$ comp 227 vendas, totalizando 705 vendas neste período de 8 meses.

Tendo mais saída o de $25 \mathrm{mg}$ sendo 549 caixas, depois o de $100 \mathrm{mg}$ sendo 133 caixas vendidas, o de $200 \mathrm{mg}$ com 16 caixas e o de $50 \mathrm{mg}$ com apenas 7 caixas vendidas.

A figura 3 demonstra as vendas em porcentagens com relação a Genéricos, Similares e de Referência da Quetiapina.

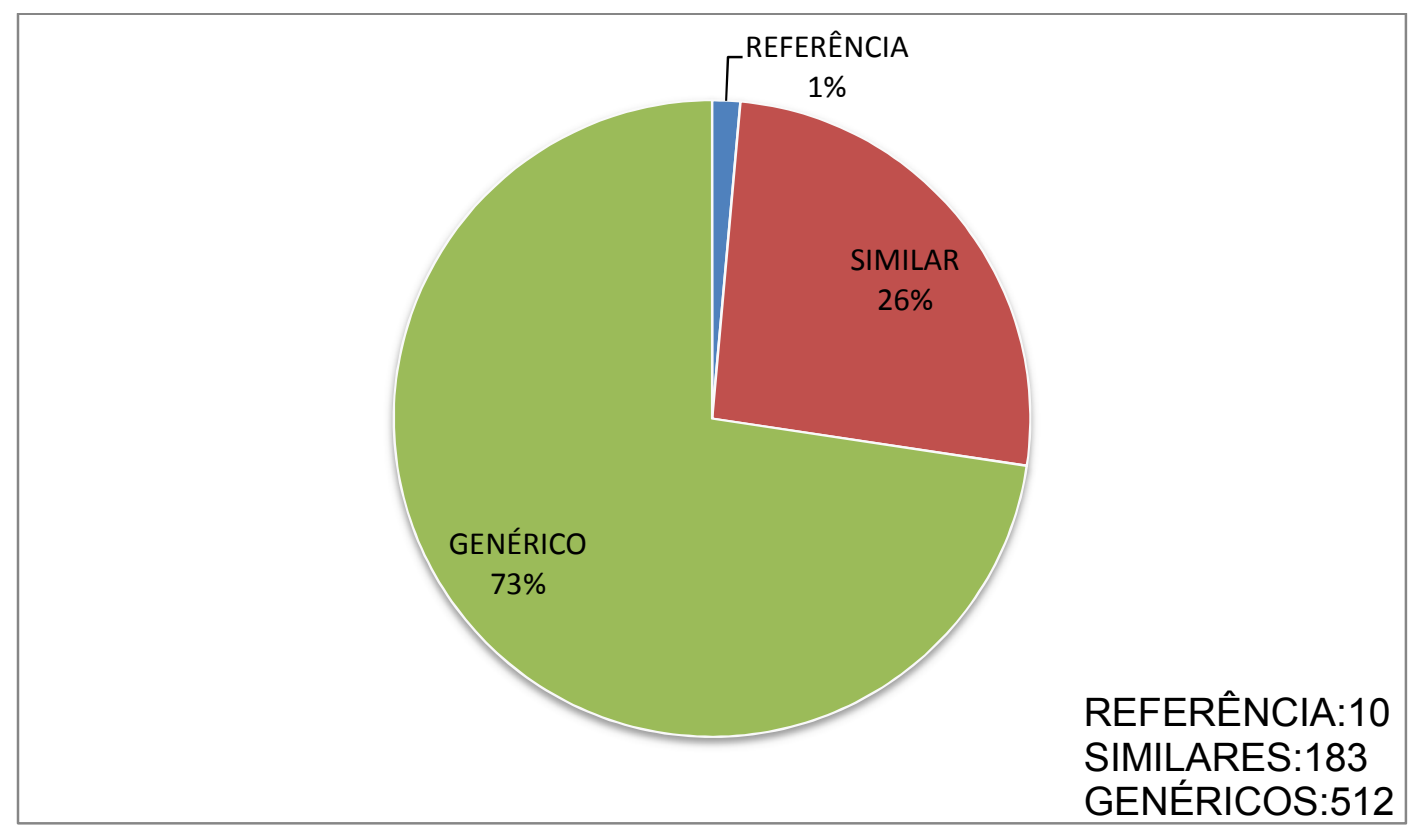

Figura 3: Vendas apresentadas em porcentagens em relação a Genéricos, Similares e de Referência da Quetiapina.

Volume 2, Número 1 - Maio, 2016. 
Em um estudo publicado pela OPAS, em 2007, em relação aos medicamentos prescritos pelas Unidades Básicas de Saúde, 78,3\% dos medicamentos são prescritos pela Relação Nacional de Medicamentos Essenciais, sendo $84,7 \%$ prescritos pelo nome Genérico.

As apresentações do fármaco genérico foram as que possuíram a maior venda $73 \%$ sendo 512 vendas, em relação aos similares $26 \%$ que foram 183 vendas e aos de Referência $1 \%$ que são apenas 10 caixas vendidas, justificada provavelmente pelo menor preço bem como a maior prescrição do fármaco pelo nome genérico. O que corrobora para este estudo, pois $73 \%$ dos medicamentos foram prescritos pelo nome genérico.

Em relação ao modelo do estudo utilizado neste trabalho, o desenho transversal, é útil para promover o uso racional de medicamentos já que os resultados obtidos podem servir de base para apoiar o uso racional de medicamentos, e para inferências em municípios de porte semelhante no Brasil.(64)

\section{CONSIDERAÇÕES FINAIS}

O Transtorno do Humor Bipolar é uma das doenças mentais mais graves que afeta cada vez mais pessoas. O fumarato de quetiapina por ser um medicamento relativamente novo demonstrou ser eficaz tanto com monoterapia ou associado à outros fármacos, bem como uma maior relação risco/benefício em comparação com outros fármacos antipsicóticos atípicos.

O perfil de comercialização do fármaco Quetiapina na drogaria demonstrou um consumo acima da média nos meses de final de ano. $E$ foram preferíveis a compra do fármaco pela apresentação genérica, seguida pela similar e posteriormente pela de referência, destes a apresentação Quetiapina Actavis $^{\circledR}$ teve a maior venda, seguida pelo Quetiapina Sandoz ${ }^{\circledR}$ e Quetros ${ }^{\circledR}$, todos de $25 \mathrm{mg}$, sugerindo a preferência das prescrições por dosagens menores do fármaco.

O Farmacêutico deve fornecer informações farmacológicas e não-farmacológicas corretas aos pacientes, familiares e outros profissionais de saúde a fim de promover o uso racional da Quetiapina no THB bem como em outras doenças, promovendo saúde e o bemestar dos pacientes.

\section{REFERÊNCIAS}

Volume 2, Número 1 - Maio, 2016. 
1. Silva AG, Bueno JR, Moreno RA. Especial transtorno bipolar. Revista debates em psiquiatria. São Paulo . 2011; 5 (1):5-5. [Acesso em: 23 set. 2014] .Disponível: http://www.abp.org.br/download/revista_debates_5.pdf.

2. Berk $L$ et al. Living with bipolar: A guide to understanding and managing the disorder. Nsw: allen and unwin. Australian. 2008; 1(1):164-176.

3. Kaplah $\mathrm{HI}$ et al. Compêndio de psiquiatria: ciências do comportamento e psiquiatria clínica. Porto Alegre: Artmed, 2003; 7 (1).

4. Miasso AL. "Entre a cruz e a espada": o significado da terapêutica medicamentosa para a pessoa com transtorno afetivo bipolar, em sua perspectiva e na de seu familiar. Ribeirão Preto. 261 f. Tese [Doutorado em Enfermagem]- Escola de Enfermagem de Ribeirão. Ribeirão Preto; 2006.

5. Shansis FM, Cordioli AV. Farmacoterapia dos Transtornos do Espectro do Humor Bipolar: diretrizes e algoritmo. Psicofármacos: consulta rápida. Porto Alegre. 2005; 1(1): 329-329.

6. Prieto E, et al. Neurobiological bases of quetiapine antidepresant effect in the bipolar disorder. Actas esp psiquiatr. Espanha. 2010; 38(1):22-32.

7. Laporte JR, Baksass I, Lunde PKM. Drug utilization studies- methods and uses. Copenhagen: regional publications european series. Berlin. 1993; 45(1): 5-22.

8. Cordeiro BC, Leite SN. O farmacêutico na atenção á saúde . 2. ed. Ed.Santa Catarina: Univalli; 2008.

9. Lieberman J. Capítulo 30. In: Alan F. Tratado psychopharmacology. Espanha: Panamericana, 2006: 517-531. 
10. Santos L, Torriani MS, Barros E. Quetiapina. Medicamentos na prática da farmácia clínica. Porto Alegre: Artmed. 2013;1 (1): 939-940.

11. Frota LH, Bueno JR, Silva Filho JF. Risperidona, amisulprida, quetiapina e ziprasidona. Comentários finais aos protocolos do ministério da saúde para antipsicóticos atípicos de segunda geração. Rio de Janeiro. 2001;1(1): 21. [Acesso em: 23 set. 2014.] Disponível em: http://www.medicina.ufrj.br/cursos/jbp risperidona, quetiapina, ziprasidona e amisulprida.pdf.

12. Almond S, O'donnell O. Cost analysis of the treatment of schizophrenia in the UK. A simulation model comparing olanzapine, risperidone and haloperidol. Canterbury. 2000; 17 (1): 383-389.

13. Hoefler R. Interações medicamentosas: Formulário Terapêutico Nacional 2008. Textos básicos de saúde. Brasília. 2008; 1(1):30-33.

14. Angst J. Historical Aspects of the Dichotomy between Manic-depressive Disorders and Schizophrenia. Schizophr res. Calgary: Alberta. 2002; 1: 5-13.

15. Pichot P. The Birth of the Bipolar Disorder. Eur psychiatry10. Londres. 1995; 1 (1): 110.

16. Marneros A. Expanding the group of bipolar disorders. J affect disord. Milano. 2001: 62 (1): 39-44.

17. Akiskal HS. L'émergence des troubles bipolaires. L'information psychiatrique. San Diego. 2005; 81(1): 857-861.

18. Del-Porto AJ, Del-Porto OK. História da caracterização nosológica do transtorno bipolar. Revista de psiquiatria clínica. São Paulo, 2005;32 (1): 7-14.

19. Quarantini LC, Sena EP, Oliveira IR. Tratamento do transtorno esquizoafetivo. Psiquiatria clínica. Salvador. 2005;1(32): 90-90. [Acesso em: 22 fev. 2015]. Disponível em: http://www.scielo.br/pdf/rpc/v32s1/24417.pdf.

Volume 2, Número 1 - Maio, 2016. 
20. Soeiro-de-Souza MG, Moreno RA, Dias VV. Guia para cuidadores de pessoas com transtorno bipolar. São Paulo: Segmento farma. 2011; 1(1): 112-112. [Acesso em: 27 ago. 2014.] Disponível em: http://www.progruda.com/UserFiles/image/ImageBank/11779GuiaBipolar1808.pdf.

21. Suppes T, Dennehy EB. Transtorno Bipolar. Porto Alegre: Marina fodra (trans.). 2009;1(1): 1-16.

22. Moreno RA, Moreno, DH, Ratzke R. Diagnóstico, tratamento e prevenção da mania e da hipomania no transtorno bipolar. Psiquiatria clínica. São Paulo. 2005: 1(32): 3948. [Acesso em: 22 fev. 2015]. Disponível em: http://www.hcnet.usp.br/ipq/revista/vol32/s1/pdf/39.pdf.

23. Pimenta MCN. Tratamento medicamentoso do transtorno bipolar de início tardio. Brasil farma. Rio de Janeiro. 2009; 3 (90): 218-220. [Acesso em: 22 fev. 2015.] Disponível em: http://www.rbfarma.org.br/files/pag_218a220_tratamento_bipolar_224.pdf.

24. Merikangas KR. et al. Prevalence and Correlates of Bipolar Spectrum Disorder in the World Mental Health Survey Initiative. Arch gen psychiatry. Bethesda. 2011; 68 (1): 241-251.

25. Merikangas, KR, Pato M. Disorder in Adults and Children: Magnitude, Correlates and Future Directions. Clinical psychology: science and practice. Bethesda. 2009;16 (1): 121-133.

26. Frey $\mathrm{BN}$, et al. Anormalidades neuropatológicas e neuroquímicas no transtorno afetivo bipolar. Revista brasileira de psiquiatria. São Paulo. 2004;1(1): 1-9.

27. Beal MF. Does impairment of energy metabolism result in excitotoxic neuronal death in neurological illnesses. Annals of neurology. Bethesda. 1992; 31(1): 119-130.

Volume 2, Número 1 - Maio, 2016. 
28. Heales SJ, et al. Nitric oxide, mitochondria and neurological disease. Biochimica et biophysica acta. London. 1999; 1410 (1): 215-228.

29. Donato S. Disorders related to mitochondrial membranes: pathology of the respiratory chain and neurodegeneration. Journal of inherited metabolic disorders. Milan. 2000;23(1): 247-263.

30. Ankarcrona M. et al. Glutamate-induced neuronal death: a succession of necrosis or apoptosis depending on mitochondrial function. Neuron, Boston. 1995; 15 (1): 961 973.

31. Pereira L. L. et al. Transtorno bipolar: reflexões sobre diagnóstico e tratamento. Perspectiva. São Paulo. 2010; 34 (128):151-166. [Acesso em: 22 fev. 2015]. Disponível em: http://www.uricer.edu.br/new/site/pdfs/perspectiva/128_144.pdf.

32. Brietzke E. et al. Impacto f comorbid magraine on clinical course of bipolar disorder. Comprehensive psychiatry. São Paulo.2011; 53 (1): 809-812.

33. Biederman J. et al. Clinical correlates of bipolar disorder in a large referred sample of children and adolescents. J psychiatr res. Boston. 2005; 39:611-622.

34. Petresco S. et al. Transtorno bipolar na infância e diagnóstico diferencial com TDAH. Casos clin psiquiatria. São Paulo. 2002; 4 (1): 54-60.

35. Barbirato F, Dias G. Transtorno de humor bipolar na infância e na adolescência. Segmento farma. Brasília. 2006; 1 (1): 1-6.

36. Carlson GA, Meyer SE. Phenomenology and diagnosis in bipolar disorder in children, adolescents and adults: complexities and developmental issues. Dev psychopathol. Nova York. 2006; 18939 (969).

Volume 2, Número 1 - Maio, 2016. 
37. Tillman $\mathbf{R}$ et al. Psychotic phenomena in 257 young children and adolescents with bipolar I disorder: delusions and hallucinations (benign and pathological). Bipolar disord. St. Louis. 2008;10 (1): 45-55.

38. Grunebaum MF, et al. Correlates of suicide attempt history in bipolar disorder: a stress-diathesis perspective. Bipolar disord. Nova York. 2006;8 (1) :551-557.

39. Gerpen MW, Johnson JE, Winstead DK. Mania in the geriatric population: a review of the literature. Am j geriatr psychiatry. Minneapolis. 2001;9(1):180-180.

40. Souza FG M. Tratamento do transtorno bipolar: Eutimia. Revista psiquiatria clínica. Fortaleza.2005;32(1): 63-70.

41. European CN. ECNP Consensus Meeting March 2000. Nice: Guidelines for investigating efficacy in bipolar disorder. Eur neuropsychopharmacol. Berlin. 2001;11 (1): 79-88.

42. Smith $L A$, et al. Effectiveness of mood stabilizers and antipsychotics in the maintenance phase of bipolar disorder: a systematic review of randomized controlled trials. Bipolar disorders. Eynsham. 2007; 9 (1): 394-412. [Acesso em: 22 fev. 2015]. Disponível em: http://onlinelibrary.wiley.com/doi/10.1111/j.1399$\underline{5618.2007 .00490 . x / a b s t r a c t .}$

43. Biel MG. et al. Continuation versus discontinuation of lithium in recurrent bipolar illness: a naturalistic study. Bipolar disorders. Nova York. 2007;9(1):435-442. [Acesso em: 22 fev. 2015]. Disponível em: http://onlinelibrary.wiley.com/doi/10.1111/j.1399-5618.2007.00389.x/abstract

44. Baldessarini RJ, et al. Decreased risk of suicides and attempts during long-term lithium treatment: a meta-analytic review. Bipolar disorders. Boston. 2006; 8(1):625639. [Acesso em: 23 fev. 2015]. Disponível em: http://onlinelibrary.wiley.com/doi/10.1111/j.1399-5618.2006.00344.x/abstract

45. Baldessarini RJ, et al. Bipolar depression: Overview and commentary. Harvard review of psychiatry. Boston. 2010: 18 (1):143-157. [Acesso em: 23 fev. 2015].

Volume 2, Número 1 - Maio, 2016. 
Disponível em:

http://journals.Iww.com/hrpjournal/Abstract/2010/04000/Bipolar_Depression_Overvie w_and_Commentary.1.aspx

46. Macedo-Soares MB, et al. Efficacy of electroconvulsive therapy in treatment-resistant bipolar disorder: a case series. J ect. Nova York. 2005; 21 (1): 31-34.

47. Yatham LN, et al. Canadian Network for Mood and Anxiety Treatments (CANMAT) guidelines for the management of patients with bipolar disorder: consensus and controversies. Bipolar Disord. Canada. 2005; 7(1): 5-69, 2005.

48. Kraguliac NV, et al. Efficacy of omega-3 fatty acids in mood disorders: a systematic review and meta-analysis. Psychopharmacology bulletin. Minneapolis. 2009; 42 (1): 39-54.

49. Colom F, Vieta E. Melhorando o desfecho do transtorno bipolar usando estratégias não farmacológicas: o papel da psicoeducação. Revista brasileira de psiquiatria. São Paulo. 2004; 26 (1): 47-50.

50. Calabrese JR, et al. A randomized double blind placebo controlled trial of quetiapine in the treatment of bipolar I and II depression. Am j psychiatry. Nova York. 2005;162(1):1351-1360.

51. Thase, M. E. et al. Efficacy of quetiapine monotherapy in bipolar I and II depression: a double- -blind, placebo-controlled study (the BOLDER II study). J clin psychopharmacol. EUA. 2006; 26 (1):600-609.

52. Goldstein, J. M. et al. Unique mechanism of action for the antidepressant properties of the atypical antipsychotic quetiapine. 20th European college of neuropsychopharmacology. Vienna.2007:1 (1);13-17, 2007.

53. Yatham, L. N. et al. Canadian Network for Mood and Anxiety Treatments (CANMAT) and International Society for Bipolar Disorders (ISBD) collaborative update of

Volume 2, Número 1 - Maio, 2016. 
CANMAT guidelines for the management of patients with bipolar disorder: update 2009. Bipolar disord. Canada. 2009; 11(1):225-255.

54. Yatham L N et al. Canadian Network for Mood and Anxiety Treatments, Canadian Network for Mood and Anxiety Treatments (CANMAT) guidelines for the management of patients with bipolar disorder: consensus and controversies. Bipolar disord. Canada. 2005; 7 (1): 5-69.

55. Suppes T et al. Trial 127 Investigators Maintenance treatment for patients with bipolar I disorder: results from a north american study of quetiapine in combination with lithium or divalproex (trial 127). Am j psychiatry. Nova York. 2009;166(1): 476-488.

56. Mckenna K, et al. Pregnancy outcome of women using atypical antipsychotic drugs: a prospective comparative study. The journal of clinical psychiatry. Nova York. 2005; 66 (1): 444-449.

57. Wozniak J, et al. Comparison of open-label, 8-week trials of olanzapine monotherapy and topiramate augmentation of olanzapine for the treatment of pediatric bipolar disorder. J child adolesc psychopharmacol. Boston. 2009;19 (1):539-545.

58. Sharma T. Quetiapine--efficacy in different domains. Eur neuropsychopharmacol. Dartford. 2001; 4(1): 385-390. [Acesso em: 23 fev. 2015]. Disponível em: http://www.europeanneuropsychopharmacology.com/article/S0924-977X(01)001122/references

59. Wong YW, YEH C, THYRUM P. T.. The effects of concomitant phenytoin administration on the steady-state pharmacokinetics of quetiapine. $\mathrm{J}$ clin psychopharmacol. Washington. 2001: 21(1): 89-93.

60. Asmal L et al. Quetiapina contra outros antipsicóticos atípicos para a esquizofrenia. Dados cochrane de revisões sistemáticas. São Paulo. 2013;11 (1):1278.

Volume 2, Número 1 - Maio, 2016. 
61. Castro C G S O. Estudos de utilização de medicamentos. Noções Básicas. Rio de Janeiro. 2000;1(1):15-25.

62. World Health Organization: Who collaborating centre for drug statistics methodology. Noruega: Oslo, 1993.

63. Castro, C. G. S. O.. Estudo de utilização de medicamentos em unidade neonatal. 1996. 34 f. [Dissertação] Mestrado - Curso de Farmácia. UFRJ. Rio de Janeiro, 1996.

64. Rozenfeld, S.; Valente, J.. Organização mundial de sáude relatório sobre a saúde no mundo saúde mental. Nova Concepção, Nova Esperança. Genebra. 2001; 1(1).

Volume 2, Número 1 - Maio, 2016. 